\title{
REDUCIBLE VON NEUMANN GEOMETRIES
}

\author{
IN MEMORY OF MAURICE AUDIN \\ BY \\ ISRAEL HALPERIN
}

1. Content of this paper. Given any irreducible von Neumann geometry $L$ and any complete Boolean algebra $B$ we shall construct a particular reducible geometry $B(L)$ which has its centre isomorphic to $B$ and is such that each Iwamura local component of $B(L)$ contains a sublattice isomorphic to $L$. If $B$ is finite or if $L$ is compact (in the dimension topology) each Iwamura local component is actually isomorphic to $L$.

We shall use a point-free generalization of the constructon given in $[2, \S 6]$. A von Neumann geometry (briefly, a geometry) $L$ is a complemented modular lattice containing at least two elements which is complete and satisfies von Neumann's lattice continuity conditions [6, pp. 1, 2, Axioms I-IV].

Our construction of $B(L)$ is valid and yields a von Neumann geometry whenever (i) $B$ is a Boolean algebra, (ii) $L$ is a complemented modular lattice, and (iii) on $L$ is given a real valued function $D(a)$ such that $0 \leqq D(a) \leqq 1$ for each $a \in L, D(0)=0, D(1)=1$, and $D(a \cup b)+D(a \cap b)=D(a)+D(b)$ for all $a, b \in L$. Thus $B(L)$ should be denoted more precisely as $B(L, D)$. However, as von Neumann showed [6, Part I, Chapter VII], if $L$ is a von Neumann geometry and irreducible (as defined in [6, p. 3, Axiom VI]) such a dimension function exists and is unique.

2. Preliminaries. As in [2] we use the function $d(a, b)=D(a \cup b)-D(a \cap b)$ defined for all $a, b \in L$. We have: $d(a, a)=0, d(a, b)=d(b, a) \geqq 0$ for all $a, b$ in $L$ (we do not exclude $d(a, b)=0$ for $a \neq b$ ). As in [5, p. 102; 1, pp. 76-77], we have, successively,

$$
\begin{aligned}
d(a \cup c, b \cup c) & +d(a \cap c, b \cap c) \\
= & D(a \cup b \cup c)-D((a \cup c) \cap(b \cup c)) \\
& +D((a \cap c) \cup(b \cup c))-D(a \cap b \cap c) \\
\leqq & D(a \cup b \cup c)-D((a \cap b) \cup c) \\
& \quad+D((a \cup b) \cap c)-D(a \cap b \cap c)
\end{aligned}
$$

(since in every lattice, $(a \cup c) \cap(b \cup c) \geqq(a \cap b) \cup c$ and $(a \cap c) \cup(b \cap c) \leqq(a \cup b) \cap c$ )

$$
=D(a \cup b)+D(c)-(D(a \cap b)+D(c))=d(a, b) ;
$$

Received by the editors April 5, 1962. 


$$
\begin{aligned}
d(a, b)+ & d(b, c) \\
& =d(a \cup b, b)+d(b, a \cap b)+d(b \cup c, b)+d(b, b \cap c) \\
& \geqq d(a \cup b \cup c, b \cup c)+d(b \cup c, b)+d(b, b \cap c)+d(b \cap c, a \cap b \cap c) \\
& =d(a \cup b \cup c, a \cap b \cap c) \\
& \geqq d(a \cup c, a \cap c)=d(a, c) ; \\
d(a \cap c, & b \cap d)+d(a \cup c, b \cup d) \\
& \leqq d(a \cap c, b \cap c)+d(b \cap c, b \cap d)+d(a \cup c, b \cup c)+d(b \cup c, b \cup d) \\
& \leqq d(a, b)+d(c, d) .
\end{aligned}
$$

3. Construction of $B(L)$. A set of nonzero disjoint elements in $B, u=\{b\}$ with supremum $=1$ will be called a partition. If $u, v$ are partitions then $u v$ denotes the partition consisting of all nonzero $b \cap c$ with $b \in u, c \in v$.

A function $f$ defined for all $b \in u$ with values in $L$ will be called a partitionfunction, more precisely, a $u$-function. If $f$ is a $u$-function and $g$ is a $v$-function then $f \cup g, f \cap g$ will denote the $u v$-functions with values $f(b) \cup g(c)$, respectively, $f(b) \cap g(c)$ for nonzero $b \cap c(b \in u, c \in v)$. We define $d(f, g)$ to be $\sup (d(f(b), g(c)) \mid b \in u, c \in v, b \cap c \neq 0)$. Clearly, if $h$ is a $w$-function then $(f \cup g) \cup h=(f \cup h) \cup(g \cup h)$.

We shall call $f=\left\{f^{n}\right\}$ fundamental (or a fundamental sequence) if each $f^{n}$ is a partition-function and $d\left(f^{n}, f^{m}\right) \rightarrow 0$ as $n, m \rightarrow \infty$. Because of the results of $\S 2$ it follows that if $f=\left\{f^{n}\right\}$ and $g=\left\{g^{n}\right\}$ are fundamental, then as $n, m \rightarrow \infty, d\left(f^{n}, g^{m}\right)$ converges to a limit, which we denote $d(f, g) ; d(f, f)=0, d(f, g)=d(g, f) \geqq 0$, $f \cup g \equiv\left\{f^{n} \cup g^{n}\right\}$ and $f \cap g=\left\{f^{n} \cap g^{n}\right\}$ are both fundamental, $d(f, g)=$ $d(f \cup g, f \cap g)$, and if $f, g, h, k$ are fundamental, then

$$
\begin{aligned}
d(f, g) & \leqq d(f, h)+d(h, g), \\
d(f \cap g, h \cap k) & \leqq d(f, h)+d(g, k), \\
d(f \cup g, h \cup k) & \leqq d(f, h)+d(g, k) .
\end{aligned}
$$

If $f, g$ are fundamental we write $f \equiv g$ if $d(f, g)=0$ and $f \leqq g$ if $d(f \cup g, g)=0$ (equivalently, $f \cup g \equiv g$ ). Clearly, $d(f, g)=d\left(f_{1}, g_{1}\right)$ if $f \equiv f_{1}$ and $g \equiv g_{1}$.

The relation $\equiv$ is easily seen to be an equivalence relation on the set of fundamental sequences; from now on we identify each fundamental sequence with its equivalence class. With this identification, we denote the set of fundamental sequences (or their equivalence classes) by $B(L)$.

Clearly $B(L)$ is ordered by the relation $f \leqq g$ defined above and $B(L)$ has a zero (namely, $f=\left\{f^{n}\right\}$ where for each $n, f^{n}(b)$ is defined for $b=1(\in B)$ with value $0(\in L)$ ), and a unit (namely $f=\left\{f^{n}\right\}$ where for each $n, f^{n}(b)$ is defined for $b=1(\in B)$ with value $1(\in L))$. 
LEMMA 1. If $f, g$ are in $L(B)$ then $f \cup g, f \cap g$ are effective as $\sup (f, g)$, $\inf (f, g)$, respectively, and $L(B)$ is a modular lattice.

Proof. $(f \cup g) \cup f=f \cup g$ so $f \cup g \geqq f$. Similarly $f \cup g \geqq g$. On the other hand, if $h$ is fundamental and $h \geqq f, h \geqq g$ hold, then because of $\S 2, d(h \cup(f \cup g), h)$ $\leqq d(h \cup f, h)+d(h \cup g, h)=0+0=0$ so $h \geqq f \cup g$. Thus $f \cup g$ is effective as $\sup (f, g)$. Similarly $f \cap g$ is effective as $\inf (f, g)$.

LEMMA 2. $L(B)$ is complemented.

Proof. Let $f$ be a given fundamental sequence. By using an equivalent fundamental sequence $\left\{f^{n}\right\}$ we may suppose (i): each $f^{n}$ is a $u_{n}$-function for partitions $u_{n}$ such that $b \in u_{n}, c \in u_{n+1}, b \cap c \neq 0$ implies $c \leqq b$, and (ii): $d\left(f^{n}, f^{n+1}\right)<1 / n^{2}$ for all $n$. Then we need only find $u_{n}$-functions $g$ for $n=1,2, \cdots$ with the properties $g^{n}(b) \cup f^{n}(b)=1, g^{n}(b) \cap f^{n}(b)=0$ for each $b \in u_{n}$ and $d\left(g^{n}, g^{n+1}\right) \leqq d\left(f^{n}, f^{n+1}\right)$. This $g=\left\{g^{n}\right\}$ is necessarily fundamental and by Lemma $1, \sup (f, g)=f \cup g=1, \inf (f, g)=f \cap g=0$, so $g$ is a complement of $f$ in $B(L)$.

Thus we need only show: for any $n \geqq 1$ and $b \in u_{n}$, given $a_{1}=f^{n}(b), a_{2}=g^{n}(b)$ with $a_{1} \cup a_{2}=1(\in L), a_{1} \cap a_{2}=0(\in L)$ and given $a_{3}=f^{n+1}(c)$ for some $c \in u_{n+1}$ with $c \leqq b$, there exists $a_{4} \in L$ such that $a_{3} \cup a_{4}=1, a_{3} \cap a_{4}=0$ and $d\left(a_{2}, a_{4}\right)$ $=d\left(a_{1}, a_{3}\right)$. For then we can define $g^{n+1}(c)=a_{4}$.

As shown in [2, Lemma 6.9], $a_{4}$ can be chosen as

$$
\left[a_{2}-\left(\left(a_{1} \cup a_{3}\right) \cap a_{2}\right)\right] \cup\left[a_{1}-\left(a_{1} \cap a_{3}\right)\right]
$$

( $[c-d]$, defined for $d \leqq c$, denotes any (fixed) relative complement of $d$ with respect to $c$ ). With this $a_{4}$,

$$
\begin{aligned}
a_{3} \cup a_{4} & =\left[a_{2}-\left(\left(a_{1} \cup a_{3}\right) \cap a_{2}\right)\right] \cup a_{1} \cup a_{3}=a_{2} \cup a_{1} \cup a_{3}=1 ; \\
a_{3} \cap a_{4} & =a_{3} \cap\left(a_{1} \cup a_{3}\right) \cap a_{4}=a_{3} \cap\left[a_{1}-\left(a_{1} \cap a_{3}\right)\right]=0 ; \\
d\left(a_{2}, a_{4}\right) & =D\left(a_{2} \cup\left[a_{1}-\left(a_{1} \cap a_{3}\right)\right]\right)-D\left(\left[a_{2}-\left(\left(a_{1} \cup a_{3}\right) \cap a_{2}\right)\right]\right) \\
& =D\left(a_{2}\right)+D\left(a_{1}\right)-D\left(a_{1} \cap a_{3}\right)-D\left(a_{2}\right)+D\left(\left(a_{1} \cup a_{3}\right) \cap a_{2}\right) \\
& =D\left(a_{1}\right)-D\left(a_{1} \cap a_{3}\right)+D\left(a_{1} \cup a_{3}\right)+D\left(a_{2}\right)-D\left(a_{1} \cup a_{3} \cup a_{2}\right) \\
& =D\left(a_{1} \cup a_{3}\right)-D\left(a_{1} \cap a_{3}\right)+1-1=d\left(a_{1}, a_{3}\right) .
\end{aligned}
$$

LEMMA 3. $L(B)$ is complete.

Proof. We need only show that for any given set of fundamental sequences $\left\{f_{\alpha} \mid \alpha \in I\right\}$, the supremum exists in $L(B)$. Since $L(B)$ is known to be a lattice we may replace $\left\{f_{\alpha} \mid a \in I\right\}$ by the set of all $\left\{f_{s} \mid s \subset I, s\right.$ finite $\}$ where $f_{s}=\bigcup\left(f_{\alpha} \mid \alpha \in s\right)$. For each $m$ we choose $m(s)$ by induction on $m$ so that $m(s)>(m-1)(s)$ and $d\left(f_{s}^{m}, f_{s}^{p}\right)<1 / m$ for all $m, p \geqq m(s)$. 
We shall now define a fundamental sequence $g=\left\{g^{n}\right\}$ and then verify that this $g$ is effective as $\sup \left\{f_{s}\right\}$. We shall define $g^{n}$ to be a $u_{n}$-function with partitions $u_{n}$ such that $b \in u_{n}, c \in u_{n+1}, b \cap c \neq 0$ imply $c \leqq b$.

Let $u_{1}$ consist of the single element 1 and set $g^{1}\left(u_{1}\right)=0(\in L)$. This defines $u_{1}$ and $g^{1}$.

Suppose for some $n>1$ that $u_{1}, \cdots, u_{n-1}, g^{1}, \cdots, g^{n-1}$ have been defined. Then for each $a \in u_{n-1}$ we shall choose certain nonzero disjoint $b \leqq a$ and define $g^{n}(b)$ for these $b$ in such a way that the set of all chosen $b$ (for all $a \in u_{n-1}$ ) will form a partition $u_{n}$ and $g^{n}$ will be a $u_{n}$-function.

We shall actually define $n$ subsets of $\{b\}$, namely $a_{n}^{1}, \cdots, a_{n}^{r}, \cdots, a_{n}^{n}$ and define $g^{n}(b)$ for $b \in a_{n}^{r}$, as follows.

Choose $a_{n}^{1}$ to be a maximal set of nonzero disjoint $b \leqq a$ such that for each $b \in a_{n}^{1}$ there exist $s, m, c$ with $m \geqq n(s), f_{s}^{m}(c)$ defined and $b \leqq c$, and $D\left(f_{s}^{m}(c)\right) \geqq 1-1 / n$, and set $g^{n}(b)=f_{s}^{m}(c)$.

If $a_{n}^{1}, \cdots, a_{n}^{r}$ have been defined for some $1 \leqq r<n$ then let

$$
\bar{a}=a-\bigcup\left(b \mid b \in a_{n}^{1} \cup \cdots \cup a_{n}^{r}\right)
$$

and choose $a_{n}^{r+1}$ to be a maximal set of nonzero disjoint $b \in B$ with $b \leqq \bar{a}$ such that for each $b \in a_{n}^{r+1}$ there exist $s, m, c$ with $m \geqq n(s), f_{s}^{m}(c)$ defined and $b \leqq c$, and $D\left(f_{s}^{m}(c)\right) \geqq 1-(r+1) / n$, and set $g^{n}(b)=f_{s}^{m}(c)$.

Clearly $u_{n}=\left\{b \mid b \in\left(a_{n}^{1} \cup \cdots \cup a_{n}^{n}\right), a \in u_{n-1}\right\}$ is a set of disjoint $b \in B$, with each $b \leqq$ some $a \in u_{n-1}$. We shall see now that $u_{n}$ has supremum in $B$ equal to 1 . Suppose, if possible that for some $a \in u_{n-1}, \sup \left(b \mid b \in\left(a_{n}^{1} \cup \cdots \cup a_{n}^{n}\right)\right)=\bar{a} \neq a$; then $a-\bar{a} \neq 0$. Then for any $s$, and any $m \geqq n(s)$ there is some $c$ for which $f_{s}^{m}(c)$ is defined and $c \cap(a-\bar{a}) \neq 0$. Then this $b=c \cap(a-\bar{a})$ could be adjoined to $a_{n}^{n}$, contradicting the maximality of $a_{n}^{n}$. Thus $\sup u_{n} \geqq a$ for every $a \in u_{n-1}$, hence $\sup u_{n}=1$, so $u_{n}$ is a partition of $B$. Clearly $g_{u}^{n}$ is a $u_{n}$-function.

Next we shall see that $\left\{g^{n}\right\}$ is fundamental. Indeed, if $g^{n}(b)$ and $g^{m}(c)$ are defined with $b \cap c \neq 0, n>m$, then $b \leqq c, D\left(g^{n}(b) \cup g^{m}(c)\right) \leqq D\left(g^{m}(c)\right)+1 / m$ since each $a_{n}^{r}$ is maximal, and $D\left(g^{n}(b)\right) \geqq D\left(g^{m}(c)\right)-1 / m \geqq D\left(g^{n}(b) \cup g^{m}(c)\right)-2 / m$. Hence

$$
\begin{aligned}
D\left(g^{n}(b) \cup g^{m}(c)\right) & -D\left(g^{n}(b) \cap g^{m}(c)\right) \\
& =2 D\left(g^{n}(b) \cup g^{m}(c)\right)-D\left(g^{n}(b)\right)-D\left(g^{m}(c)\right) \\
& \leqq \frac{1}{m}+\frac{2}{m}=\frac{3}{m} .
\end{aligned}
$$

So $d\left(g^{n}, g^{m}\right) \rightarrow 0$ as $n, m \rightarrow \infty$. This means that $g=\left\{g^{n}\right\}$ is fundamental.

Next we show that $g \geqq f_{s}$ for each $s$. We need only show that $d\left(g \cup f_{s}, g\right)=0$; hence we need only show that $d\left(g^{m} \cup f_{s}^{m(s)}, g^{m}\right) \rightarrow 0$ as $m \rightarrow \infty$.

Suppose $g^{m}(b)$ and $f_{s}^{m(s)}(c)$ are defined for some $b \cap c \neq 0$. Then 
$D\left(f_{s}^{m(s)}(c) \cup g^{m}(b)\right) \leqq D\left(g^{m}(b)\right)+2 / m$ so $d\left(g^{m} \cup f_{s}^{m(s)}, g^{m}\right) \leqq 2 / m \quad$ and $\rightarrow 0$ as $m \rightarrow \infty$, as required.

Finally, we shall show that if $h$ is fundamental and $h \geqq f_{s}$ for all $s$ then $h \geqq g$. Suppose $h=\left\{h^{n}\right\}$ and choose $n(h)$ so that $d\left(h^{m}, h^{p}\right)<1 / n$ for all $m, p \geqq n(h)$. Then $d\left(h^{m} \cup f_{s}^{p}, h^{m}\right) \leqq 2 / n$ if $m \geqq n(h)$ and $p \geqq n(s)$. Hence $d\left(h^{m} \cup g^{n}, h^{m}\right) \leqq 2 / n$ if $m \geqq n(h)$ since each $g^{n}(b)=f_{s}^{p}(c)$ for some $s$ and some $p \geqq n(s)$ and some $c \geqq b$. Hence $d\left(h^{m} \cup g^{m}, h^{m}\right) \rightarrow 0$ as $m \rightarrow \infty$. Thus $h \geqq g$ as required.

This completes the proof that $B(L)$ is complete.

LEMMA 4. $L(B)$ is ascending-continuous.

Proof. We need to show that if $f_{\alpha} \leqq f_{\beta}$ for all $\alpha<\beta<\Omega$ for some limit ordinal $\Omega$, then $f \cap \bigcup\left(f_{\alpha} \mid \alpha<\Omega\right)=\bigcup\left(f \cap f_{\alpha} \mid \alpha<\Omega\right)$. Since $L(B)$ is complemented and modular it is sufficient to show that if $f \cap f_{\alpha}=0$ for each $\alpha$ then $f \cap \bigcup\left(f_{\alpha} \mid \alpha \in I\right)=0$.

Let $g=\bigcup\left(f_{\alpha} \mid \alpha \in I\right)$ be formed as in Lemma 3. Then since $f \cap f_{\alpha}=0$, it follows for every $\alpha$ that $D\left(f^{m}(b) \cap f_{\alpha}^{m}(c)\right)<2 / n$ if $m \geqq n(f)$ and $m \geqq n(\alpha)$, where $n(f)$ is chosen so that $d\left(f^{m}, f^{p}\right)<1 / n$ if $m, p \geqq n(f)$, and $n(\alpha)$ is chosen so that $d\left(f_{\alpha}^{m}, f_{\alpha}^{p}\right)<1 / n$ if $m, p \geqq n(\alpha)$, and $b, c$ are elements $\in B$ such that $f^{m}(b), f_{\alpha}^{m}(c)$ are defined and $b \cap c \neq 0$.

Now if $g^{n}(a)$ is defined then $g^{n}(a)=f_{\alpha}^{m}(c)$ for some $\alpha$, some $m \geqq n(\alpha)$ and some $c$ for which $a \leqq c$ and $f_{\alpha}^{m}(c)$ is defined.

Hence $d\left(f^{m} \cap g^{m}, 0\right)<2 / n$ if $m \geqq n(f)$ and $m \geqq n$, and so $\rightarrow 0$ as $m \rightarrow \infty$. Hence $d(f \cap g, 0)=0$ so $f \cap g=0$. This proves Lemma 4 .

LEMMA 5. $L(B)$ is descending-continuous.

Proof. If $\left\{f_{\alpha} \mid \alpha \in I\right\}$ are given, we form $g=\bigcap\left(f_{\alpha} \mid \alpha \in I\right)$ by a procedure dual to that used in Lemma 3. Then Lemma 5 can be verified by an argument dual to that used in Lemma 4.

THEOREM 1. $L(B)$ is a von Neumann geometry.

Proof. This is a restatement of Lemmas 1-5.

REMARK 1. If $L$ satisfies a chain condition, that is, $L$ is the direct sum of a finite number of discrete ( $=$ finite dimensional irreducible projective) geometries then the proof of Theorem 1 can be simplified; in this case $B(L)$ coincides with the set of all partition functions.

REMARK 2. If $D(a)>0$ for $a \neq 0, B(L)$ contains a sublattice $L$ which is lattice isomorphic to $L$ namely the constant sequences $f_{a}=\left\{f_{a}^{n}\right\}$ with $f_{a}^{n}(b)$ defined for $b=1$ and $f_{a}^{n}(1)=a \in L$, for all $n$. The mapping $f_{a} \leftrightarrow a$ is a lattice isomorphism.

REMARK 3. Let $B_{0}$ be the Boolean algebra consisting of 0,1 only and suppose $D(a)>0$ if $a \neq 0$, so $B_{0}(L)$ is a von Neumann geometry containing $\bar{L}$ (isomorphic to $L$ ) as a sublattice. Then $B_{0}(L)=\bar{L}$ if and only if $L$ is a von Neumann geometry and $D\left(\bigcup\left(a_{\alpha} \mid \alpha<\Omega\right)=\sup \left(D\left(a_{\alpha}\right) \mid \alpha<\Omega\right)\right.$ whenever $a_{\alpha} \leqq a_{\beta}$ for all $\alpha \leqq \beta<\Omega$. 
Remark 4. If $L$ is a von Neumann geometry and $D(a)>0$ for $a \neq 0$ and $D\left(\bigcup\left(a_{\alpha} \mid \alpha<\Omega\right)\right)=\sup \left(D\left(a_{\alpha}\right) \mid \alpha<\Omega\right)$ whenever $a_{\alpha} \leqq a_{\beta}$ for all $\alpha \leqq \beta<\Omega$, then $Z$ the centre of $L$ is a complete Boolean algebra isomorphic to a finite measure algebra (then $Z$ cannot contain a noncountable set of nonzero disjoint elements).

Remark 5. We could construct $B(L)$ in terms of an arbitrary family of given dimension functions $D_{i}$ (in place of one function $D$ ) but then to obtain a von Neumann geometry $B(L)$ we would need to use fundamental filters $f$ (in place of fundamental sequences). We omit the details.

We shall call a fundamental sequence $\left\{f^{n}\right\}$ distributive if whenever $f^{n}(b)$ is defined its value is distributive in $L(z \in L$ is called distributive in $L$ if $z \cap(a \cup b)=(z \cap a) \cup(z \cap b)$ for all $a, b \in L)$. It is clear that the distributive fundamental sequences form a sublattice of $B(L)$ which is isomorphic to (and henceforth will be identified with) $B(Z) \equiv B(D, Z)$ where $Z$ is the Boolean algebra of all distributive elements in $L$ (in any complemented modular lattice, the set of distributive elements coincides with the centre of the lattice).

Lemma 6. $f$ is distributive in $B(L)$ if $(*) f$ is equivalent to an element in $B(Z)$. If $L$ is a von Neumann geometry then $f$ is distributive in $B(L)$ if and only if $(*)$ holds; the centre of $B(L)$ is $B(Z)$.

Proof. The first part of Lemma 6 follows from the definitions of $f \cup g, f \cap g$.

On the other hand, if $f=\left\{f^{n}\right\}$ is distributive in $B(L)$ then for each $n$ let $q_{n}=\sup \left\{\inf \left\{d\left(f^{n}(b), z\right) \mid z \in Z\right\} \mid\right.$ all $b$ for which $f^{n}(b)$ is defined $\}$, let $z(b) \in Z$ be chosen so that $d\left(f^{n}(b), z(b)\right)<q_{n}+1 / n$ and let $f^{n}(b)$ be defined, $=z(b)$ for each $b$ for which $f^{n}(b)$ is defined.

If $q_{n} \rightarrow 0$ as $n \rightarrow \infty$ it will follow that $\tilde{f}=\left\{\bar{f}^{n}\right\}$ is fundamental, $\in B(Z)$ and $f \equiv \bar{f}$, as desired.

Thus to prove Lemma 6, we may assume that for some fixed $\varepsilon>0: q_{n}>\varepsilon$ for an infinite number of $n$ and we need only derive a contradiction (assuming that $L$ is a von Neumann geometry). Now for some $n_{0}$, and for some $b_{0}$ for which $f^{n_{0}}\left(b_{0}\right)$ is defined: (i) $d\left(f^{n_{0}}\left(b_{0}\right), z\right)>\varepsilon$ for all $z \in Z$ and (ii) for all $n \geqq n_{0}$ the $c_{n}$ for which $f^{n}\left(c_{n}\right)$ is defined and $c_{n} \cap b_{0} \neq 0$ satisfy $d\left(f^{n}\left(c_{n}\right), f^{n_{0}}\left(b_{0}\right)\right)<\varepsilon / 4$.

But for any $a, c$ in $L$, the constant sequences $g=\left\{g^{n}\right\}, h=\left\{h^{n}\right\}$ with $g^{n}(1)=a, h^{n}(1)=c$ for all $n$, are fundamental; since $f$ is distributive in $B(L)$ hence $d((f \cap(g \cup h)),(f \cap g) \cup(f \cap h))=0$, so

$$
d\left(\left(f^{n}\left(c_{n}\right) \cap(a \cup c)\right),\left(\left(f^{n}\left(c_{n}\right) \cap a\right) \cup\left(f^{n}\left(c_{n}\right) \cap c\right)\right)\right) \rightarrow 0
$$

as $n \rightarrow \infty$. Hence

$$
d\left(f^{n_{0}}\left(b_{0}\right) \cap(a \cup c), \quad\left(f^{n_{0}}\left(b_{0}\right) \cap a\right) \cup\left(f^{n_{0}}\left(b_{0}\right) \cap c\right)\right)<\frac{\varepsilon}{2} .
$$

Thus to obtain the desired contradiction we need only show: if $b \in L$ with $d(b, z)>\varepsilon$ for all $z \in Z$ then there exists $a, c \in L$ such that 


$$
d(b \cap(a \cup c),(b \cap a) \cup(b \cap c))>\frac{\varepsilon}{2} .
$$

This is shown in the following lemma, which completes the proof of Lemma 6.

Lemma 7. Suppose that $L$ is a von Neumann geometry with a dimension function $D(a)$ such that $0 \leqq D(a) \leqq 1$ for all $a \in L, D(0)=0, D(1)=1$ and $D(a \cup b)+D(a \cap b)=D(a)+D(b)$ for all $a, b$ in $L$. Then for any $b$ in $L$,

$$
\inf \{(D(b \cup z)-D(b \cap z)) \mid z \in Z\} \leqq D(b \cap(a \cup c))-D((b \cap a) \cup(b \cap c))
$$

for some $a, c \in L$.

Proof. It is sufficient to exhibit $a, c \in L, z_{0} \in Z$ such that

$$
D\left(b \cup z_{0}\right)-D\left(b \cap z_{0}\right)=D(b \cap(a \cup c))-D((b \cap a) \cup(b \cap c)) .
$$

For this purpose let $h$ be any complement in $L$ of $b$. Apply von Neumann [6, Part III, Theorem 2.7] to $b$ and $h$. Then there exist disjoint elements $e_{1}, e_{2} \in Z$ such that

$$
\begin{aligned}
& b=b_{1} \cup b_{2} \text { with } b_{1}=b \cap e_{1}, b_{2}=b \cap e_{2}, \\
& h=h_{1} \cup h_{2} \text { with } h_{1}=h \cap e_{1}, h_{2}=h \cap e_{2},
\end{aligned}
$$

and $h_{1}$ is perspective to some $\bar{b}_{1} \leqq b_{1}$ and $b_{2}$ is perspective to some $h_{2} \leqq h_{2}$. Hence for suitable $x, y$ in $L$,

$$
\begin{array}{ll}
h_{1} \cup x=\bar{b}_{1} \cup x=h_{1} \cup \bar{b}_{1} ; & h_{1} \cap x=b_{1} \cap x=0 ; \\
h_{2} \cup y=b_{2} \cup y=h_{2} \cup b_{2} ; & h_{2} \cap y=b_{2} \cap y=0 .
\end{array}
$$

Set $a=h_{1} \cup h_{2}, c=x \cup y$. Then

$$
\begin{aligned}
D(b \cap(a \cup c))-D((b \cap a) \cup(b \cap c)) \\
\quad=D\left(b \cap\left(h_{1} \cup h_{2} \cup x \cup y\right)\right)-D\left(\left(b \cap\left(h_{1} \cup h_{2}\right)\right) \cup(b \cap(x \cup y))\right) \\
\quad=D\left(b_{1} \cap\left(h_{1} \cup x\right)\right)+D\left(b_{2} \cap\left(h_{2} \cup y\right)\right)-0 \\
=D\left(\bar{b}_{1}\right)+D\left(b_{2}\right)=D\left(h_{1}\right)+D\left(b_{2}\right) \\
=D\left(e_{1}\right)-D\left(b_{1}\right)+D\left(b_{2}\right) \\
=D\left(b \cup e_{1}\right)-D\left(b \cap e_{1}\right) .
\end{aligned}
$$

Since $e_{1}$ is in Z, Lemma 7 (and hence also Lemma 6) is established.

THEOREM 2. If $L$ is an irreducible von Neumann geometry and $B$ is a complete Boolean algebra, the centre of $B(L)$ is isomorphic to $B$.

Proof. Suppose that $b \in B$ and let $f_{b}=\left\{f_{b}^{n}\right\}$ be the constant sequence with $f_{b}^{n}(b)$, $f_{b}^{n}(1-b)$ defined, $=1,0$, respectively, for all $n$. Then the mapping $b \leftrightarrow f_{b}$ is clearly 
a $(1,1)$ lattice isomorphism of $B$ onto a sublattice of the centre of $B(L)$ since $d\left(f_{b}, f_{c}\right)=D(1) \neq 0$ if $b \neq c$.

On the other hand, suppose $f$ is in the centre of $B(L)$. We may suppose $f=\left\{f^{n}\right\}$ with $\left\{f^{n}\right\}$ a distributive sequence. Now for any $n$, if $f^{n}(b)$ is defined, we must have $f^{n}(b)=0$ or 1 since $L$ has centre consisting of 0,1 only. Let $b^{n}=\bigcup\left(b \mid f^{n}(b)=1\right)$. Then $d\left(f^{n}, f^{m}\right) \geqq D(1)$ if $b^{n} \neq b^{m}$. Hence for some $n_{0}, b^{n}$ is constant, $=b$ say, for all $n \geqq n_{0}$ and $f$ is equivalent to the constant sequence $f_{b}$.

This proves Theorem 2 .

4. The Iwamura local components of $B(L)$. Iwamura [3; 4], defined local components for any von Neumann geometry $L$ with centre $Z$ in (essentially) the following way.

First, write: $|f| \leqq 1 / r$ to mean that $r$ is a positive integer, $f \in L$ and there exist independent elements $f=f_{1}, f_{2}, \cdots, f_{r}$ in $L$ such that each $f_{i}$ is perspective to $f$.

Next, let $p$ be a maximal dual ideal of $Z$. This means: $p \subset Z$ and $p$ has the following properties:

(i) $b \in p, c \in Z, c \geqq b$ together imply $c \in p$;

(ii) $b \in p, c \in p$ together imply $b \cap c \in p$;

(iii) $0 \notin p$;

(iv) $p$ is maximal with properties (i), (ii), (iii).

Next, define $f \leqq g$ (at $p$ ) to mean: $f, g \in L$ and for any relative complement $h=[(f \cup g)-g]$, and for each integer $r>0$, there exists some $b_{r}$ in $p$ such that $\left|b_{r} \cap h\right| \leqq 1 / r$. Define $f \equiv g$ (at $p$ ) to mean: $f \leqq g$ (at $p$ ) and $g \leqq f($ at $p$ ) both hold.

Then as Iwamura showed, the relation " $\equiv($ at $p)$ " is an equivalence relation in $L$ and if $f$ is identified with its equivalence class, the set of equivalence classes is ordered under the relation " $\leqq($ at $p)$ " and is an irreducible von Neumann geometry, denoted $L / p$. We shall call $L / p$ the Iwamura local component of $L$ at $p$.

Throughout the rest of this section we shall assume that $L$ is an irreducible von Neumann geometry, that $D$ denotes its (unique) dimension function, that $B$ is a complete Boolean algebra and that $p$ is a maximal dual ideal of $B$. For each $c \in L, f_{c}=\left\{f_{c}^{n}\right\}$ will denote the constant sequence with $f_{c}^{n}(1)$ defined and equal to $c$ for all $n$.

We shall prove:

THEOREM 3. If $L$ is an irreducible von Neumann geometry and $B$ is a complete Boolean algebra then $L$ is isomorphic to the subgeometry of all $f_{c}$ in $B(L)($ at $p)$ under the mapping $c \rightarrow f_{c}$.

Lemma 8. With the hypotheses of Theorem 3 , if $f_{a} \leqq f_{c}$ (at p) then $a \leqq c$.

Proof. Let $e=[(a \cup c)-c]$. Then $f_{e}=\left[\left(f_{a} \cup f_{c}\right)-f_{c}\right]$. For every integer $r>0$ there exists some $b \in p$ for which $\left|b \cap f_{e}\right|<1 / r$. This means: there are 
elements $f_{1}=b \cap f_{e}, f_{2}, \cdots, f_{r}$ independent in $B(L)$ and elements $g_{2}, \cdots, g_{r}$ in $B(L)$ such that $g_{j} \cup f_{1}=g_{j} \cup f_{j}=1, g_{j} \cap f_{1}=g_{j} \cap f_{j}=0$ (in $B(L)$ ) for $j \geqq 2$.

We may, without loss of generality, suppose that for each $n$ there is a partition $u_{n}$ and $f_{j}=\left\{f_{j}^{n}\right\}, g_{j}=\left\{g_{j}^{n}\right\}$ with $u_{n}$-functions $f_{j}^{n}$ and $g_{j}^{n}$.

For each $n$ choose an element $b_{n} \in u_{n}$. Then as $n \rightarrow \infty$ :

$$
\begin{aligned}
& D\left(g_{j}^{n}\left(b_{n}\right) \cup e\right) \rightarrow 1, D\left(g_{j}^{n}\left(b_{n}\right) \cup f_{j}^{n}\left(b_{n}\right)\right) \rightarrow 1, \\
& D\left(g_{j}^{n}\left(b_{n}\right) \cap e\right) \rightarrow 0, D\left(g_{j}^{n}\left(b_{n}\right) \cap f_{j}^{n}\left(b_{n}\right)\right) \rightarrow 0,
\end{aligned}
$$

and for $2 \leqq j \leqq r$,

$$
D\left(f_{j}^{n}\left(b_{n}\right) \cap\left(\bigcup_{i=2}^{i-1} f_{i}^{n}\left(b_{n}\right) \cup e\right)\right) \rightarrow 0
$$

It follows that for $2 \leqq j \leqq r$,

$$
D\left(f_{j}^{n}\left(b_{n}\right)\right) \rightarrow D(e) \text { as } n \rightarrow \infty,
$$

and

$$
D\left(e \cup f_{2}^{n}\left(b_{n}\right) \cup \cdots \cup f_{r}^{n}\left(b_{n}\right)\right) \rightarrow r D(e) \text { as } n \rightarrow \infty .
$$

Since $D(a) \leqq 1$ for all $a \in L, r D(e) \leqq 1, D(e) \leqq 1 / r$.

Since $D(e) \leqq 1 / r$ for all $r>0, e=0$ and so $a \leqq c$ as stated.

Proof of Theorem 3. Obviously $a \leqq b$ implies $f_{a} \leqq f_{b}$ (at $p$ ); Lemma 8 shows that $f_{a} \leqq f_{b}$ (at $p$ ) implies $a \leqq b$. It follows that the mapping $c \rightarrow f_{c}$ is an isomorphism of $L$ and the subgeometry of $B(L) / p$ consisting of the elements of the form $f_{c}$.

We shall call an irreducible von Neumann geometry $L$ compact if for each integer $n$ there exist a finite set of elements $a_{1}, \cdots, a_{m} \in L(m=m(n))$ such that for each $b \in L: \min \left(D\left(a_{i} \cup b\right)-D\left(a_{i} \cap b\right) \mid i=1, \cdots, m\right)<1 / n$; in other words, $L$ is compact in the metric topology

$$
d(a, b)=D(a \cup b)-D(a \cap b) .
$$

It is easy to see that $L$ is compact if and only if for each real number $\alpha$ $(0 \leqq \alpha \leqq 1)$ the set of elements of dimension $\alpha$ is compact (or empty); if $L$ is a discrete (that is, projective finite dimensional) geometry, this condition is equivalent to: the number of elements in $L$ is finite.

We shall prove:

THEOREM 4. If $L$ is an irreducible von Neumann geometry and $B$ is a complete Boolean algebra, the Iwamura local components of $B(L)$ are all isomorphic to $L$ if either the number of elements in $B$ is finite or $L$ is compact.

Lemma 9 (Von Neumann [5, p. 107]). Every irreducible von Neumann geometry Lis a complete metric space under the metric: $d(a, b)=D-(a \cup b)-D(a \cap b)$. 
Proof. Suppose that $d\left(a_{n}, a_{m}\right) \rightarrow 0$ as $n, m \rightarrow \infty$. Choose a subsequence $a_{n(m)}$, with $n(m)<n(m+1)$ for all $m=1,2, \cdots$ such that $d\left(a_{n(m)}, a_{n(m+1)}\right)<1 / m^{2}$. Then $a=\bigcap_{m=1}^{\infty}\left(\bigcup_{r=m}^{\infty} a_{n(r)}\right)$ satisfies: $d\left(a, a_{n(m)}\right) \rightarrow 0$ as $m \rightarrow \infty$ and hence $d\left(a, a_{n}\right) \rightarrow 0$ as $n \rightarrow \infty$.

LEMMA 10. Suppose that $L$ is an irreducible von Neumann geometry and $a, b_{1}, c \in L$ with $b_{1} \leqq a \cup c, a \cap c=b_{1} \cap c=a \cap b_{1}=0$ and $D(a)=D(c)$. Then for some $b \geqq b_{1}: a \cup b=b \cup c=a \cup c$ and $a \cap b=b \cap c=0$.

Proof. Let $a_{1}=a \cap\left(b_{1} \cup c\right), c_{1}=c \cap\left(b_{1} \cup a\right)$. Then

$$
\begin{aligned}
a_{1} \cup b_{1} & =\left(a \cup b_{1}\right) \cap\left(c \cup b_{1}\right)=c_{1} \cup b_{1} \\
& =a_{1} \cup c_{1}, a_{1} \cap b_{1}=b_{1} \cap c_{1}=c_{1} \cap a_{1}=0 .
\end{aligned}
$$

Now let $a_{2}=\left[a-a_{1}\right], c_{2}=\left[c-c_{1}\right]$. Then $D\left(a_{2}\right)=D(a)-D\left(a_{1}\right)=D(c)-D\left(c_{1}\right)$ $=D\left(c_{2}\right), a_{2} \cap c_{2}=0$ and $\left(a_{2} \cup c_{2}\right) \cap\left(a_{1} \cup c_{1}\right)=0$. Hence for some $b_{2} \leqq a_{2} \cup c_{2}$ we have $a_{2} \cup b_{2}=c_{2} \cup b_{2}=a_{2} \cup c_{2}$ and $a_{2} \cap b_{2}=c_{2} \cap b_{2}=0$.

It is easily seen that $b=b_{1} \cup b_{2}$ satisfies the requirements of Lemma 10 .

LEMMA 11. Suppose that Lis an irreducible von Neumann geometry, $B$ is a Boolean algebra, $f=\left\{f^{n}\right\}$ is in $B(L)$, and for some $r \geqq 2, D\left(f^{n}(b)\right) \leqq 1 / r$ for all $n$ and $b$ for which $f^{n}(b)$ is defined. Then $|f| \leqq 1 / r$.

Proof. We may suppose each $f^{n}$ is a $u_{n}$-function with partitions $u_{n}$ such that $b \in u_{n}, c \in u_{n+1}, b \cap c \neq 0$ imply $c \leqq b$. By using a suitable subsequence of the $f^{n}$ we may also suppose that $\sum_{n=1}^{\infty} d\left(f^{n}, f^{n+1}\right)<\infty$. Denote $f$ as $f_{1}$.

We shall show that for some fixed constant $k<\infty$, we can define, for each $n$ and each $b \in u_{n}$, values in $L, f_{j}^{n}(b), g_{j}^{n}(b), j=2, \cdots, r$ so that

$$
f_{1}^{n}(b), \cdots, f_{r}^{n}(b) \text { are independent in } L,
$$

$$
\begin{aligned}
& g_{j}^{n}(b) \cup f_{1}^{n}(b)=g_{j}^{n}(b) \cup f_{j}^{n}(b)=f_{1}^{n}(b) \cup f_{j}^{n}(b), \\
& g_{j}^{n}(b) \cap f_{1}^{n}(b)=g_{j}^{n}(b) \cap f_{j}^{n}(b)=0,
\end{aligned}
$$

and so that if $n>1$ and $b \in u_{n-1}, c \in u_{n}$ and $c \leqq b$, then

$$
\begin{aligned}
& d\left(g_{j}^{n-1}(b), g_{j}^{n}(c)\right) \leqq k d\left(f^{n}, f^{n+1}\right), \\
& d\left(f_{j}^{n-1}(b), f_{j}^{n}(c)\right) \leqq k d\left(f^{n}, f^{n+1}\right) .
\end{aligned}
$$

It will follow that $g_{j}=\left\{g_{j}^{n}\right\}, f_{j}=\left\{f_{j}^{n}\right\}, j=2, \cdots, r$, are all in $B(L)$, that $f_{1}, \cdots, f_{r}$ are independent in $B(L)$ and for $j \geqq 2, f_{j}$ is perspective to $f_{1}$. This will show that $|f| \leqq 1 / r$ as required.

We define $f_{j}^{n}(b), g_{j}^{n}(b)$ for all $b \in u_{n}$ by induction on $n$. For $n=1$, we choose arbitrarily $f_{j}^{1}(b), g_{j}^{1}(b)$ for all $b \in u_{1}$, to satisfy $\left(\mathrm{P}_{1}\right)$. This is possible since $D\left(f_{1}^{1}(b)\right) \leqq 1 / r$ for all $b \in u_{1}$. 
Now suppose for some $n \geqq 1$ that $g_{j}^{n}(b), f_{j}^{n}(b)$ are defined for all $b \in u_{n}$ and satisfy $\left(\mathrm{P}_{n}\right)$. Then we define $g_{j}^{n+1}(c), f_{j}^{n+1}(c)$ for all $c \in u_{n+1}$, and $j=2, \cdots, s, \cdots, r$ by induction on $s$ as follows, so as to satisfy $\left(\mathrm{P}_{n+1}\right)$ and $\left(\mathrm{Q}_{n+1}\right)$.

Let $\bar{g}_{2}^{n+1}(c)=g_{2}^{n}(b) \cap f_{1}^{n+1}(c)$. If $\left.D\left(g_{2}^{n} b\right)\right)-D\left(\bar{g}_{2}^{n+1}(c)\right) \geqq D\left(f_{1}^{n+1}(c)\right)$ choose $g_{2}^{n+1}(c)$ arbitrarily $\leqq g_{2}^{n}(b)$ but with $g_{2}^{n+1}(c) \cap f_{1}^{n+1}(c)=0$ and

$$
D\left(g_{2}^{n+1}(c)=D\left(f_{1}^{n+1}(c)\right)\right. \text {. }
$$

Otherwise, choose $g_{2}^{n+1}(c)=\left[g_{2}^{n}(b)-\bar{g}_{2}^{n+1}(c)\right] \cup h_{2}^{n+1}(c)$ where $h_{2}^{n+1}(c)$ is any element in $L$ with $h_{2}^{n+1}(c) \cap\left(g_{2}^{n}(b) \cup f_{1}^{n+1}(c)\right)=0$ and $D\left(g_{2}^{n+1}(c)\right)=D\left(f_{1}^{n+1}(c)\right)$. Such an element $h_{2}^{n+1}(c)$ exists since in the present case, $D\left(g_{2}^{n}(b) \cup f_{1}^{n+1}(c)\right)$ $\leqq 2 D\left(f_{1}^{n+1}(c)\right)$.

Now $g_{2}^{n+1}(c) \cap f_{1}^{n+1}(c)=0$. Let $f_{2}^{n+1}(c)=f_{2}^{n}(b) \cap\left(g_{2}^{n+1}(c) \cup f_{1}^{n+1}(c)\right)$ and let $\left(\bar{f}_{2}^{\prime}\right)^{n+1}(c)=\left(\bar{f}_{2}^{n+1}(c) \cap g_{2}^{n+1}(c)\right) \cup\left(\bar{f}_{2}^{n+1}(c) \cap f_{1}^{n+1}(c)\right)$.

Then $\left.\left.D\left(\bar{f}_{2}^{n+1}\right)(c)\right)-D\left(\bar{f}_{2}^{\prime}\right)^{n+1}(c)\right) \leqq D\left(f_{1}^{n+1}(c)\right)$. Choose

$$
\left.f_{2}^{n+1}(c) \leqq g_{2}^{n+1}(c) \cup f_{1}^{n+1}(c) \text { and } \geqq{ }_{\llcorner} \tilde{f}_{2}^{-n+1}(c)-\left(f_{2}^{\prime}\right)^{n+1}(c)\right]
$$

and so that

$$
f_{2}^{n+1}(c) \cap g_{2}^{n+1}(c)=f_{2}^{n+1}(c) \cap f_{1}^{n+1}(c)=0
$$

and $f_{2}^{n+1}(c) \cup g_{2}^{n+1}(c)=f_{1}^{n+1}(c) \cup g_{2}^{n+1}(c)=f_{2}^{n+1}(c) \cup f_{1}^{n+1}(c)$. This is possible because of Lemma 10.

Now suppose for some $s=2, \cdots, r-1$ that $f_{j}^{n+1}(c), g_{j}^{n+1}(c)$ have been defined for all $2 \leqq j \leqq s$ so that: $f_{1}^{n+1}(c), \cdots, f_{s}^{n+1}(c)$ are independent, $f_{1}^{n+1}(c) \cup g_{j}^{n+1}(c)$ $=f_{j}^{n+1}(c) \cup g_{j}^{n+1}(c)=f_{1}^{n+1}(c) \cup g_{j}^{n+1}(c)$ and $f_{1}^{n+1}(c) \cap g_{j}^{n+1}(c)=f_{j}^{n+1}(c) \cap g_{j}^{n+1}(c)$ $=0$. Then define $g_{s+1}^{n+1}(c), f_{s+1}^{n+1}(c)$ as follows.

Let $\bar{g}_{s+1}^{n+1}(c)=g_{s+1}^{n}(b) \cap\left(f_{1}^{n+1}(c) \cup \cdots \cup f_{s}^{n+1}(c)\right)$. If $D\left(g_{s+1}^{n}(b)\right)-D\left(\bar{g}_{s+1}^{n+1}(c)\right)$ $\geqq D\left(f_{1}^{+1}(c)\right)$, choose $g_{s+1}^{n+1}(c) \leqq g_{2}^{n}(b)$ but with $g_{s+1}^{n+1}(c) \cap \bar{g}_{s+1}^{n+1}(c)=0$ and $D\left(g_{s+1}^{n+1}(c)\right)=D\left(f_{1}^{n+1}(c)\right)$. Otherwise, choose

$$
g_{s+1}^{n+1}(c)=\left[g_{s+1}^{n}(b)-\bar{g}_{s+1}^{n+1}(c)\right] \cup h_{s+1}^{n+1}(c)
$$

with $h_{s+1}^{n+1}(c) \cap\left(g_{s+1}^{n}(b) \cup f_{1}^{n+1}(c) \cup \cdots \cup f_{s}^{n+1}(c)\right)=0$ and $D\left(g_{s+1}^{n+1}(c)\right)=D\left(f_{1}^{n+1}(c)\right)$. Such an element $h_{s+1}^{n+1}(c)$ exists since in the present case,

$$
D\left(g_{s+1}^{n}(b) \cup f_{1}^{n+1}(c) \cup \cdots \cup f_{s}^{n+1}(c)\right) \leqq(s+1) / r \leqq 1 .
$$

Now $\left\{g_{s+1}^{n+1}(c), f_{1}^{n+1}(c), \cdots, f_{s}^{n+1}(c)\right\}$ are independent in $L$. Let $f_{s+1}^{n+1}(c)=f_{s+1}^{n}(b)$ $\cap\left(g_{s+1}^{n+1}(c) \cup f_{1}^{n+1}(c)\right)$ and let $\left(\tilde{f}_{s+1}^{\prime}\right)^{n+1}(c)=\left(\bar{f}_{s+1}^{n+1}(c) \cap g_{s+1}^{n+1}(c)\right) \cup\left(\tilde{f}_{s+1}^{n+1}(c) \cap f_{1}^{n+1}(c)\right.$.

Then $D\left(\bar{f}_{s+1}^{n+1}(c)\right)-D\left(\left(\bar{f}_{s+1}^{\prime}\right)^{n+1}(c)\right) \leqq D\left(f_{1}^{n+1}(c)\right)$. Choose $f_{s+1}^{n+1}(c) \leqq g_{s+1}^{n+1}(c) \cup$ $f_{1}^{n+1}(c)$ and $\geqq\left[\bar{f}_{s+1}^{n+1}(c)-\left(\bar{f}_{s+1}^{\prime}\right)^{n+1}(c)\right]$ and so that $f_{s+1}^{n+1}(c) \cap g_{s+1}^{n+1}(c)=f_{s+1}^{n+1}(c)$ $\cap f_{1}^{n+1}(c)=0$ and $f_{s+1}^{n+1}(c) \cup g_{s+1}^{n+1}(c)=f_{1}^{n+1}(c) \cup g_{s+1}^{n+1}(c)=f_{s+1}^{n+1}(c) \cup f_{1}^{n+1}(c)$.

This is possible because of Lemma 10 . 
Now by induction on $s,\left(\mathrm{P}_{n+1}\right)$ will be satisfied. As for $\left(\mathrm{Q}_{n+1}\right)$, we calculate: either $d\left(g_{2}^{n}(b), g_{2}^{n+1}(c)\right) \leqq d\left(f_{1}^{n}(b), f_{1}^{n+1}(c)\right)$ or (since $\left.D\left(\bar{g}_{2}^{n+1}(c)\right) \leqq d\left(f_{1}^{n}(b), f_{1}^{+1}(c)\right)\right)$ $d\left(g_{2}^{n}(b), g_{2}^{n+1}(c)\right) \leqq 2 d\left(f_{1}^{n}(b), f_{1}^{n+1}(c)\right)$.

Next,

$$
\begin{aligned}
D\left(f_{2}^{n}(b)\right)-D\left(f_{2}^{n+1}(c)\right) & \leqq d\left(g_{2}^{n}(b), g_{2}^{n+1}(c)\right)+d\left(f_{1}^{n}(b), f_{1}^{n+1}(c)\right) \\
& \leqq 3 d\left(f_{1}^{n}(b), f_{1}^{n+1}(c)\right), \\
D\left(\left(\bar{f}_{2}^{\prime}\right)^{n+1}(c)\right) & \leqq 3 d\left(f_{1}^{n}(b), f_{1}^{n+1}(c)\right),
\end{aligned}
$$

hence $d\left(f_{2}^{n}(b), f_{2}^{n+1}(c)\right) \leqq 12 d\left(f_{1}^{n}(b), f_{1}^{n+1}(c)\right)$.

Similarly it can be verified that $\left(\mathrm{Q}_{n+1}\right)$ holds for some constant $k$ which is independent of $n$.

Thus Lemma 11 holds.

LEMMA 12. With the hypotheses of Theorem 4 suppose that $f=\left\{f^{n}\right\}$ is in $B(L), p$ is a maximal dual ideal of $B$, and for an infinite number of $n: f^{n}$ is a a $u_{n}$-function and some $b_{n} \in u_{n}$ satisfies $b_{n} \in p$. Then for some $c \in L, f \equiv f_{c}$ (at $\left.p\right)$.

Proof. Replacing $\left\{f^{n}\right\}$ by an equivalent sequence we may suppose that for each $n: f^{n}$ is a $u_{n}$-function and $b_{n} \in u_{n}, b_{n} \in p$ hold.

Since $d\left(f^{n}\left(b_{n}\right), f^{m}\left(b_{m}\right)\right) \rightarrow 0$ as $n, m \rightarrow \infty$ it follows from Lemma 9 that $d\left(f^{n}\left(b_{n}\right), c\right) \rightarrow 0$ as $n \rightarrow \infty$ for some $c \in L$.

We shall prove, with this element $c$, that $f \equiv f_{c}$ (at $p$ ). As in the proof of Lemma 2 we may suppose that $g^{n}=\left[\left(f^{n} \cup f_{c}^{n}\right)-\left(f^{n} \cap f_{c}^{n}\right)\right]$ is chosen in such a way that $g=\left\{g^{n}\right\}$ satisfies $g=\left[\left(f \cup f_{c}\right)-\left(f \cap f_{c}\right)\right]$ in $B(L)$.

To show that $f \equiv f_{c}$ (at $p$ ) is equivalent to showing: for each $r>0$ there exists some $b \in p$ for which $|g \cap b|<1 / r$.

Choose a fixed $s$ so that $D\left(g^{s}\left(b_{s}\right)\right)<1 / 2 r$ and $d\left(g^{s}, g^{n}\right)<1 / 2 r$ for $n \geqq s$. Then $b_{s} \cap g=\left\{b_{s} \cap g^{n+s}\right\}$ satisfies the hypothesis of Lemma 11 , hence $\left|b_{s} \cap g\right|<1 / r$. This proves Lemma 12.

COROLlary. If $b_{0}$ is an atom in $B$ and $p$ consists of all $b \in B$ with $b \geqq b_{0}$ then $B(L) / p$ is isomorphic to $L$ under the mapping $c \rightarrow f_{c}$ of $L$ onto the subgeometry of all $f_{c}$ in $B(L)$ (at $\left.p\right)$.

Proof. Assume given $f=\left\{f^{n}\right\}$ in $B(L)$ with $f^{n}$ a $u_{n}$-function. Then for some (unique) $b_{n} \in u_{n}, b_{n} \geqq b_{0}$ and hence $b_{n} \in p$. Lemma 12 applies and shows that $f \equiv f_{c}$ for some $c \in L$. The Corollary now follows from Theorem 3 .

Proof of Theorem 4. If $B$ has only a finite number of elements, every maximal dual ideal $p$ is determined by some atom, as in the hypothesis of the corollary to Lemma 12 , and for this case, Theorem 4 follows from the corollary to Lemma 12.

Suppose now that $L$ is compact and for each $n$, choose $a_{1}, \cdots, a_{m}(m=m(n))$ 
in $L$ so that for each $c$ in $L, d\left(a_{i}, c\right)<1 / n$ for at least one $i$. Given any $f=\left\{f^{n}\right\}$ in $B(L)$ with $f^{n}$ a $u_{n}$-function set, for each $n$,

$b_{i}^{n}=\bigcup\left(b \mid f^{n}(b)\right.$ is defined and $i$ is the first index for which $\left.d\left(a_{i}, f^{n}(b)\right)<1 / n\right)$ and define $g=\left\{g^{n}\right\}$ by $g^{n}\left(b_{i}^{n}\right)=a_{i}(i=1, \cdots, m)$. It is easy to see that $g=f$ in $B(L)$ and for every maximal dual ideal $p$ and each $n$, one of the $b_{i}^{n} \in p$. Hence Lemma 12 applies andfor the case of compact $L$, Theorem 4 follows from Theorem 3.

This completes the proof of Theorem 4 .

\section{REFERENCES}

1. Garrett Birkhoff, Lattice theory, Amer. Math. Soc. Colloq. Publ. Vol. 25, Amer. Math. Soc., Providence, R. I., 1948.

2. Israel Halperin, Dimensionality in reducible geometries, Ann. of Math. 40 (1939), 581-599.

3. Tsurane Iwamura, On continuous geometries. I, Japan. J. Math. 19 (1944), 57-71.

4. Fumitomo Maeda, Kontinuierliche Geometrien, Springer, Berlin, 1958.

5. John von Neumann, Continuous geometry and examples of continuous geometries, Proc. Nat. Acad. Sci. U. S. A. 22 (1936), 92-108.

6. ——, Continuous geometry, Princeton Univ. Press, Princeton, N. J., 1960.

QUEEN'S UNIVERSTTY, Kingston, Canada 